$11-2005$

\title{
Measurement of Interfacial Shear Mechanical Properties in Thermal Barrier Coating Systems by a Barb Pullout Method
}

\author{
S. Q. Guo \\ National Institute for Materials Science, GUO.shuqi@nims.go.jp \\ D. R. Mumm \\ University of California - Irvine
}

Anette M. Karlsson

Cleveland State University, a.karlsson@csuohio.edu

Y. Kagawa

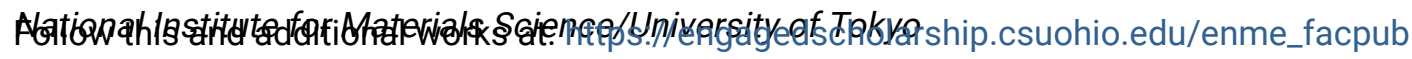

Part of the Mechanical Engineering Commons

How does access to this work benefit you? Let us know!

\section{Publisher's Statement}

NOTICE: this is the author's version of a work that was accepted for publication in Scripta

Materialia. Changes resulting from the publishing process, such as peer review, editing, corrections, structural formatting, and other quality control mechanisms may not be reflected in this document. Changes may have been made to this work since it was submitted for publication. A definitive version was subsequently published in Scripta Materialia, 53, 9, (11-01-2005); 10.1016/j.scriptamat.2005.07.012

\section{Original Citation}

Guo, S. Q., Mumm, D. R., Karlsson, A. M., 2005, "Measurement of Interfacial Shear Mechanical Properties in Thermal Barrier Coating Systems by a Barb Pullout Method," Scripta Materialia, 53(9) pp. 1043-1048.

This Article is brought to you for free and open access by the Mechanical Engineering Department at EngagedScholarship@CSU. It has been accepted for inclusion in Mechanical Engineering Faculty Publications by an authorized administrator of EngagedScholarship@CSU. For more information, please contact library.es@csuohio.edu. 


\title{
Measurement of interfacial shear mechanical properties in thermal barrier coating systems by a barb pullout method
}

\author{
S.Q. Guo ${ }^{\mathrm{a}, *}$, D.R. Mumm ${ }^{\text {b }}$, A.M. Karlsson ${ }^{\mathrm{c}}$, Y. Kagawa ${ }^{\mathrm{a}, \mathrm{d}, \mathrm{e}, *}$ \\ ${ }^{a}$ National Institute for Materials Science, 1-2-1 Sengen, Tsukuba, Ibaraki 305-0047, Japan \\ ${ }^{\mathrm{b}}$ Chemical Engineering and Materials Science, University of California, Irvine, CA 92697-2575, USA \\ c Mechanical Engineering, University of Delaware, Newark, DE 19716-3140, USA \\ ' Department of Materials Engineering, The University of Tokyo, 7-3-1 Hongo, Bunkyu-ku, Tokyo 113-8586, Japan \\ ' Institute of Industrial Science, The University of Tokyo, 4-6-1 Komaba, Meguro-ku, Tokyo 153-8505, Japan
}

\section{Introduction}

Thermal barrier coatings (TBCs) are enabling materials in the design of advanced gas turbine engine systems $[1,2]$. Based on ceramics with low thermal conductivity, such coatings provide thermal insulation to actively cooled metallic components, allowing designers to optimize system performance by maximizing combustion temperatures. Given the role of TBCs in shielding structural components from damaging thermal environments, coating durability is of primary importance. Thus, understanding the mechanisms that dictate coating failure has been the subject of intense research. It is well recognized that TBCs exhibit several failure mechanisms

\footnotetext{
- Corresponding authors. Tel.: +81 29859 2223; fax: +81 29859 2401 (S.Q. Guo); Tel.: +81 35452 5086; fax: +81354525087 (Y. Kagawa).

E-mail addresses: GUO.shuqi@nims.go.jp (S.Q. Guo), kagawa@ iis.u-tokyo.ac.jp (Y. Kagawa).
}

[3-12], and that these mechanisms often have competing dependencies on constituent material properties. In general, coating failure is governed by a series of events that include crack nucleation, propagation and coalescence, leading to coating spallation. Coating durability is then dependent upon the interplay between the crack driving forces and the resistance to crack propagation through the coating or along the relevant interfaces.

Experimental measurements of crack growth resistance are needed to refine and validate models of coating failure processes, and to determine the linkages between delamination resistance and the relevant chemical and microstructural parameters. Direct measurements of coating and/or interfacial toughness in TBC systems are recent and sparse [13-15]. The focus here is on facilitating such experimental measurements. A key concern is to accurately simulate the loading conditions that drive failure in TBCs, as crack growth resistance is highly dependent upon the mode-mixity of loading. Additionally, the toughness of a TBC is also highly 
anisotropic, depending strongly on the coating deposition technique and the resulting microstructure. For failures caused by large compressive stresses that develop within the coating, delamination crack growth occurs under principally mode II loading-particularly for longer cracks with fully-developed crack wake contact zones. Hence, the durability or lifetime is a direct function of mode II toughness of the coating.

A number of test approaches have been developed for measuring key toughness parameters in thin film and coating systems [13-17]. These test methodologies rely on inducing film delamination in a controlled manner, allowing for a quantitative measurement of adhesion or crack resistance. However, each approach has limitations, both experimentally and in application to a particular materials performance issue. Examples of test approaches that have been developed for ceramic coating systems include: (1) blister or bulge propagation methods [16] that rely on pressurization from the underside of the film or coating; (2) bend tests of notched multilayer beams [15,16]; and (3) indentation techniques $[13,16,17]$ that depend upon developing plastic strains in a ductile substrate and transmitting those strains to the coating. Each of these test methodologies is appropriate over a particular range of mode-mixities. For example, blister growth methods typically result in opening mode crack growth, with phase angles in the range of $\psi ; 0-30^{\circ}$. Bending methods are only appropriate for mixed-mode cracking measurements, where the applied loading is equally opening and shear (phase angles of $\psi ; 40-45^{\circ}$ ). The impression methods, typified by conical and wedge indentation, result in phase angles that approach $\psi=90^{\circ}$ as the delamination front moves away from the indenter $[14,18]$.

However, other challenges and limitations exist in using these techniques for evaluation of TBC failure. For example, the blister tests are hampered by the need for special sample preparation approaches (back-thinning of wafers), whereas the indentation experiments, although much more appropriate for evaluation of shear mechanical properties, are highly dependent upon the plastic properties of the substrate [18] and are very sensitive to the anisotropic (porous) structure of the ceramic top coat [19]. In this work, we describe a test that obviates many of the issues with existing protocols for measuring the fracture resistance of TBCs and related multi-layer systems. The methodology is based on a 'barb' geometry, and originates from similar approaches applied in the study of fiber reinforced ceramic and/or metal matrix composites $[20,21]$. The approach is particularly useful in capturing crack growth behavior under mode II loading conditions, and is amenable to measurement of both short-crack and long-crack behavior. It allows direct measurement of the relative opening and shear displacements, and direct correlation of crack length, applied load and microstructural aspects. The technique is illustrated by investigating the interfacial shear mechanical properties of an electron-beam physical vapor deposition (EB-PVD) TBC system. The barb test is demonstrated to have utility in investigating the delamination behavior of this coating.

\section{Materials and experimental techniques}

To demonstrate the utility of the test technique, an EB-PVD TBC system was investigated in this study. The EB-PVD TBC system was supplied by the JFCC (Japan Fine Ceramics Center, Nagoya, Japan). This system consisted of $4 \mathrm{~mol} \% \mathrm{Y}_{2} \mathrm{O}_{3}$ stabilized $\mathrm{ZrO}_{2}$, deposited by physical vapor deposition onto the metallic substrate to a thickness of $\sim 200 \mu \mathrm{m}$. The substrate was a MA738LC nickel-based superalloy, over-coated with a NiCoCrAlY bond coat layer, and sectioned into pieces averaging $55 \mathrm{~mm} \times 20 \mathrm{~mm} \times 2.5 \mathrm{~mm}$ in size. The bond coat was deposited by plasma spray deposition to a thickness of $100-150 \mu \mathrm{m}$, and had an overall composition of 32 wt. $\%$ Ni, 21 wt. $\%$ Cr, 8 wt.\% Al, 0.5 wt.\% Y, with the balance Co. The bond coat surface was then polished up to 1200 grit before the TBC deposition. Fig. 1(a) shows a typical columnar structure observed in the EBPVD TBC layer. A thermally grown oxide (TGO) layer, $0.5 \mu \mathrm{m}$ thick, is present between the TBC layer and the bond coat after processing. High-magnification images revealed the undulated nature of the bond coat/TGO interface, as shown in Fig. 1(b) (indicated by arrows).
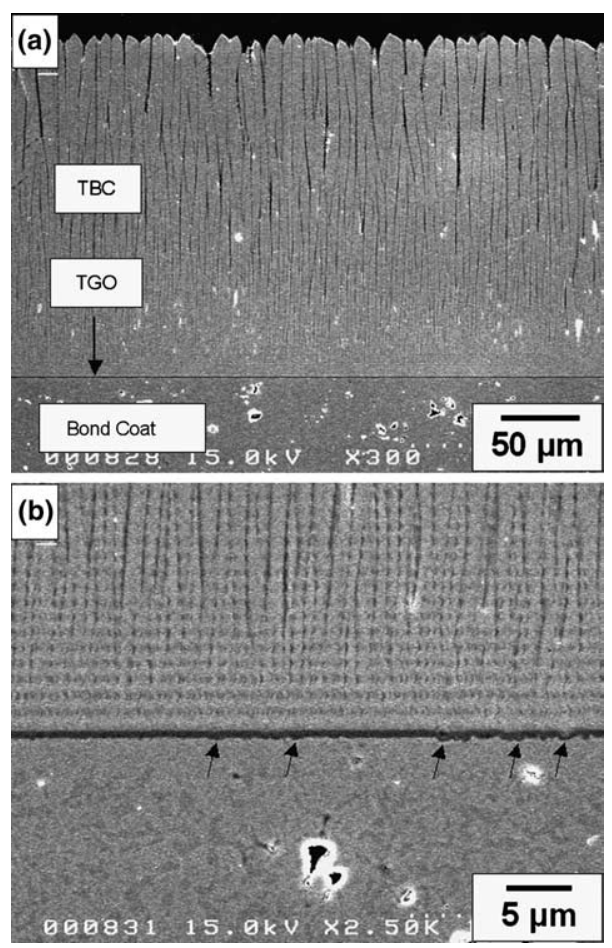

Fig. 1. (a) A typical columnar structure of the EB-PVD TBC, and (b) a high-magnification image showing the undulated nature of the bond coat/TGO interface. 
(a) Front View

(b) Side View
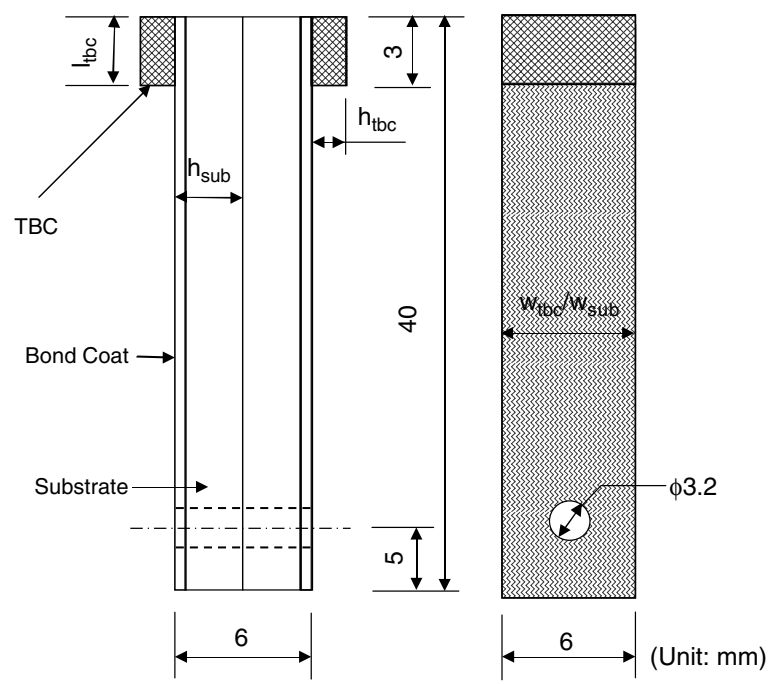

Fig. 2. Shape and dimensions of the specimen for barb pullout testing.

A schematic of the barb test specimen is shown in Fig. 2, illustrating the overall shape and specific dimensions used in this study. Test specimens with dimensions of $40 \mathrm{~mm} \times 6 \mathrm{~mm} \times 3 \mathrm{~mm}$ were cut from the EB-PVD-processed TBC specimens, using a conventional mechanical cutting procedure. The cut faces were ground through standard metallurgical procedures to (1) obtain specimens with the desired final thickness, (2) introduce parallel surfaces, and (3) eliminate mechanical flaws introduced during the cutting process. The TBC layers were then notched at a distance of $3 \mathrm{~mm}$ from the end of the specimens, to define the length over which crack growth occurs. The remaining TBC segments were carefully removed with a WC polishing tool. Two specimen pieces prepared in this manner were then affixed back-to-back, using an alignment tool and epoxy adhesive, to form the final barb test specimens.

A schematic illustration of the test fixture for barb pullout method is shown in Fig. 3. The fixture is designed to provide flat surfaces that transmit force to the cut edge of the TBC layer during relative translation of the metallic substrate. Given that the ceramic coating layers are generally very thin ( $200 \mu \mathrm{m})$, ensuring proper contact with the test fixture is a primary concern of the test technique. In the present study, sapphire plates that could be translated normal to the specimen surface were used as load transfer or support blocks. Prior to performing the barb test, the position of the sapphire supports were adjusted using the fine-thread adjustment pins in the loading fixtures. The sapphire was positioned to provide optimal load transfer to the cut edge of the coating, free of a frictional load transfer to the metallic substrate.

The barb tests were performed in ambient air at room temperature using a screw-driven mechanical test system

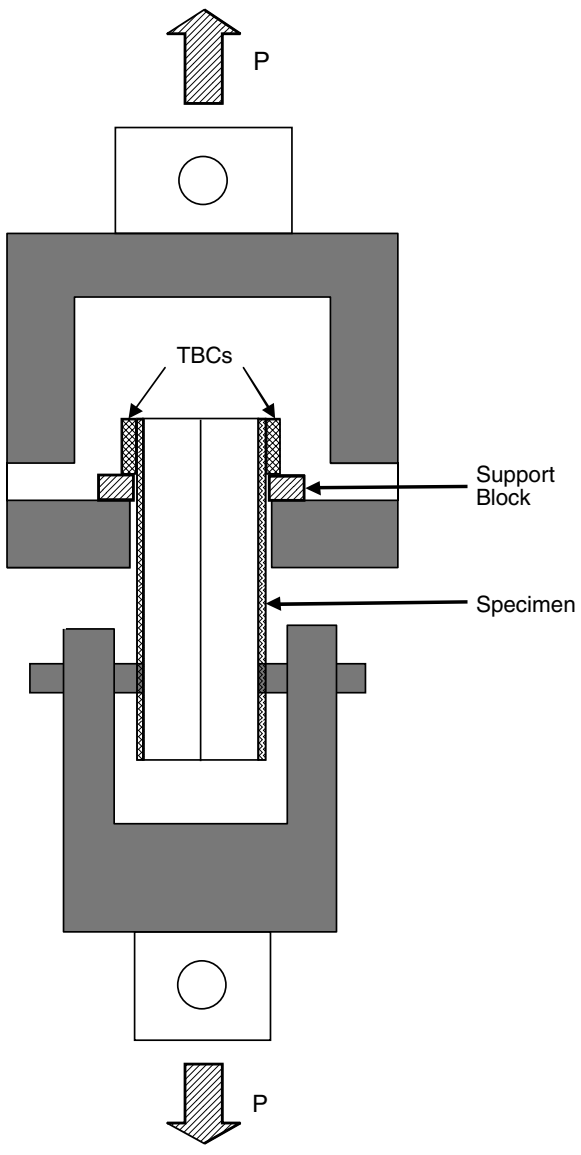

Fig. 3. Schematic illustration of the loading fixture utilized for the barb pullout testing.

(Model Autograph AG-500E, Shimadzu Corp., Kyoto, Japan). Testing was performed with a constant cross head displacement rate of $0.1 \mathrm{~mm} / \mathrm{min}$. The forcedisplacement response during the testing was digitized and continuously recorded using a digital memory scope (ORM 1200, Yokogawa Electric, Tokyo, Japan). Five specimens were tested. After test completion, the specimens were examined using scanning electron microscopy (SEM) to investigate the crack path selection, and general morphology of the fracture surfaces.

\section{Measurements and observations}

\subsection{Force-displacement curve}

A typical plot of response force versus crosshead displacement $(P-u)$, obtained from barb testing of the EB-PVD TBC specimen, is shown in Fig. 4. The force increases nearly linearly up to a maximum force, with the exception of an initial nonlinear segment corresponding to compliance associated with the test fixture and specimen alignment in the self-aligning mechanisms. The maximum force, $P_{\max }$, corresponds to the point at which the applied force is sufficient to initiate delamination of the 


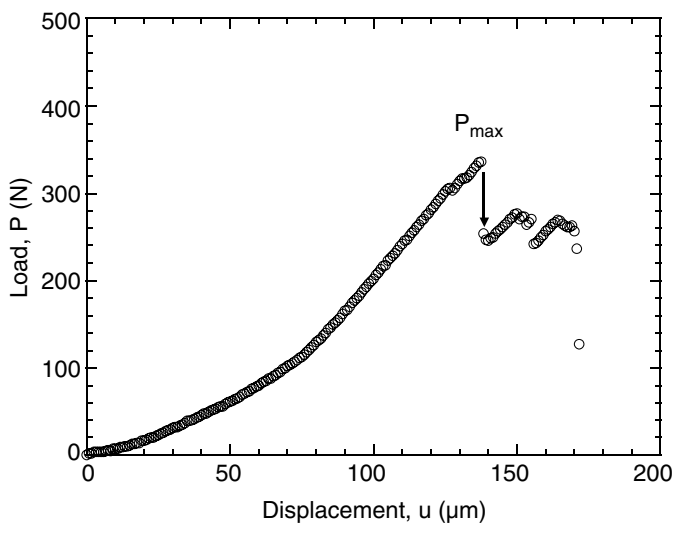

Fig. 4. A typical force-displacement curve obtained during the barb pullout testing for the EB-PVD TBC system.

TBC layer from the underlying substrate. Direct observations with a long-standoff distance optical microscope show no visible delamination crack growth prior to the peak force point. Three or four instantaneous force drops are typically observed after the maximum force, $P_{\max }$, but prior to complete debonding. This behavior suggests that propagation of the interfacial delamination occurs incrementally. Direct observation shows the coatings on both sides of the sample remain intact, adhered to the substrate over part of the contact area after the initial force drops. Complete decohesion coincides with the final drop to zero response force. The measured force drop corresponds to direct observation of complete delamination over the full contact length.

\subsection{Fracture surface observations}

SEM micrographs of the exposed metallic substrate side of fracture surface after TBC delamination are shown in Fig. 5. The low-magnification images illustrate the complex crack growth behavior under shear loading mode. The failure surface was a mixture of exposed metallic bond coat and regions of TGO (some with a remaining thin, adherent TBC layer) (Fig. 5(a)). Highresolution images show more clearly the fractured, embedded segments of TGO and the thin TBC layer bonded to these TGO particles (Fig. 5(b)). The presence of a thin TBC layer observed on the fracture surface is not fully understood, but is linked to the heterogeneous structure of the TBC coatings. A thin layer (thickness

$0.1 \mu \mathrm{m}$ ) of the TBC material, consisting of equiaxed grains of $30 \mathrm{~nm}$ diameter and having much higher density, is often observed at the boundary between the columnar TBC structure and the TGO layer [22]. The properties of this layer are sufficiently different from the columnar layers that the preferred crack path during delamination is often through the TBC layer above, leaving the thin equiaxed TBC layer on the bond coat side of the exposed fracture surface. Despite the presence of these adherent oxides, the fracture surface
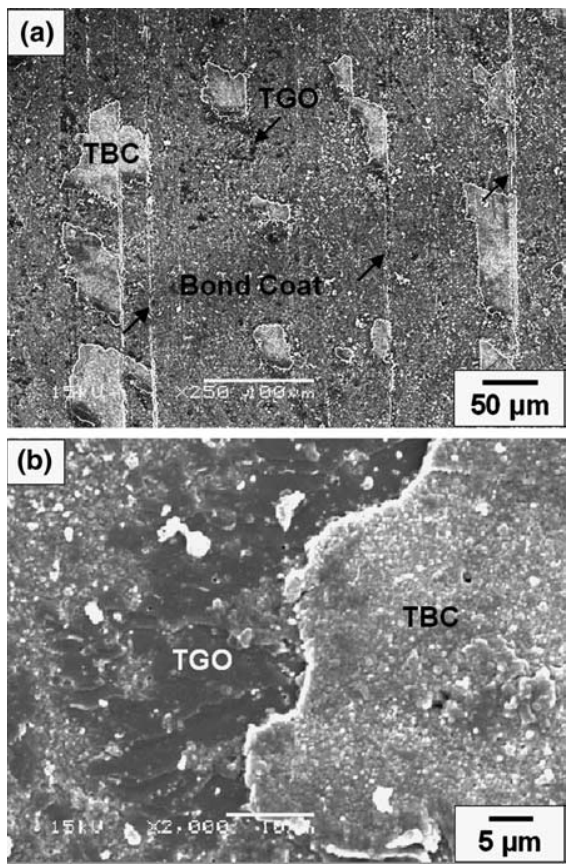

Fig. 5. SEM micrographs of the fracture surface on the metallic substrate side exposed by the delamination of the TBC layer in the EB-PVD TBC system: (a) a low-magnification image revealing characteristics of the crack growth and failure surface and (b) a high-magnification image showing embedded thermally grown oxides in the bond coat and the existence of thin layers of TBC remaining adhered to the TGO segments.

remained relatively smooth, flat relative to any relevant microstructural length scale (Fig. 5(a)). The nature of the failure observed is typical for an EB-PVD TBC system, where failure occurs along a mixed pathway through $\mathrm{TGO} /$ bond coat or TBC/TGO interfaces and/ or bulk TBC which consists of equiaxed grains nearby the TBC/TGO interface.

On the other hand, wear tracks, indicating a sliding along the spalling interface during the testing, are observed on the exposed fracture surface (examples are indicated by arrows in Fig. 5(a)). The wear tracks observed on the failure surfaces evidence that the crack faces remained in contact during the crack advance, and that wake frictional effects act to stabilize growth of the delamination of TBC layer. This stabilization likely accounts for the apparent incremental crack growth observed after peak force. Additionally, examination of the underside of delaminated TBC reveals that it is principally TBC with some patches of TGO, as shown in Fig. 6(a). High-magnification images, exemplified in Fig. 6(b), reveal the presence of micropores in the TGO layer. This view is taken from close to the edge of one of the spalled segments of TBC, such that the TBC columns may also be observed. From the above observations, it is apparent that delamination proceeded essentially at the TGO layer, with the crack propagating along either the TGO/TBC interface or the TGO/bond 

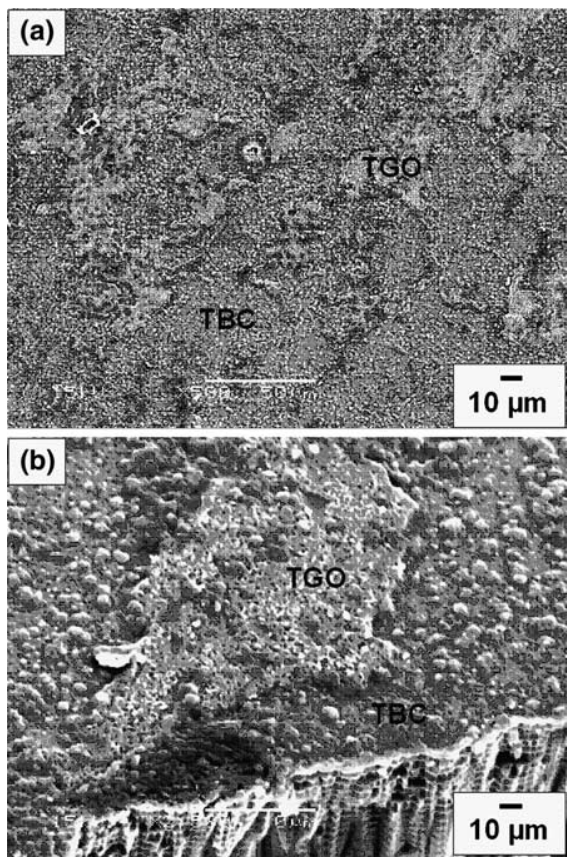

Fig. 6. SEM micrographs of the underside of the delaminated TBC layer in the EB-PVD TBC system: (a) a low-magnification image showing a surface of TBC with patches of attached TGO, and (b) a high-magnification image showing micropores in the attached TGO as well as columnar structure of the TBC layer.

coat interface, and periodically deviating into the TBC layer for the EB-PVD TBC system studied here. Based on these observations, the delamination behavior is schematically illustrated in Fig. 7.

(a) As-Coated TBCs

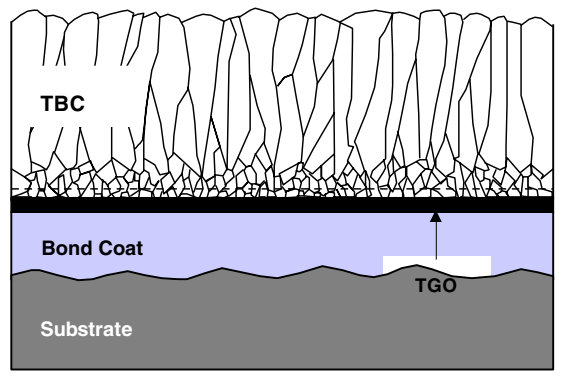

(b) Post-Tested TBCs

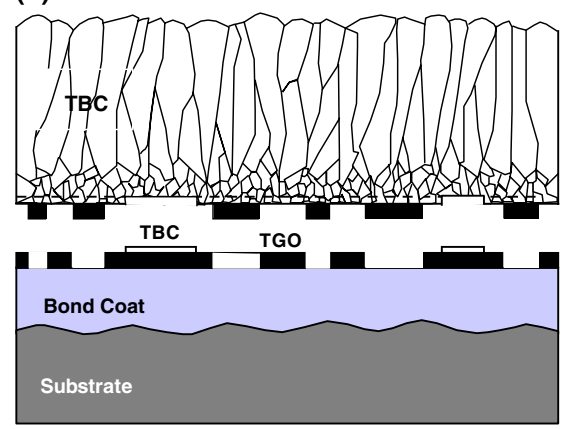

Fig. 7. Schematic illustrations showing the delamination behavior of a TBC layer during the barb pullout testing for the EB-PVD TBC system: (a) as-coated and (b) post-tested.

\section{Analysis}

To quantify the effective coating/interface toughness, the delamination strain energy release rate should be determined. For the test configuration used here, the thickness of the substrate, $h_{\text {sub }}$, is much greater than that of the TBC layer, $h_{\mathrm{tbc}}\left(h_{\mathrm{sub}} \gg h_{\mathrm{tbc}}\right)$, and the length, $l_{\mathrm{tbc}}$, is also much larger than the thickness of the TBC, $\left(l_{\mathrm{tbc}} \gg h_{\mathrm{tbc}}\right)$. Fig. 4 indicates that the failure occurs more or less instantaneously. Under such conditions, an estimate of the strain energy release rate, $G_{i}$, is obtained by assuming (1) that all of the strain energy stored in the TBC layer and the substrate prior to initiating delamination is released in developing new surface area during crack propagation, and (2) that the delamination is uniform along the crack tip without buckling. Neglecting the anisotropic nature of the $\mathrm{TBC}$ elastic properties, the strain energy release rate is approximated as [23]:

$G_{i} \approx \frac{h_{\mathrm{sub}} \sigma_{\mathrm{sub}}^{2}}{E_{\mathrm{sub}}}+\frac{h_{\mathrm{tbc}} \sigma_{\mathrm{tbc}}^{2}}{E_{\mathrm{tbc}}}$

where $\sigma_{\text {sub }}$ and $\sigma_{\text {tbc }}$ are the uniaxial stress in the substrate and TBC layer, respectively, $E_{\mathrm{sub}}$ and $E_{\mathrm{tbc}}$ are Young's modulus of the substrate and the TBC layer, respectively. Young's modulus is taken to be $E_{\text {sub }}=$ $200 \mathrm{GPa}$ for the substrate [24] and $E_{\mathrm{tbc}}=44 \mathrm{GPa}$ for the EB-PVD TBC [13]. By way of a force balance, the in-plane stress in the coatings, due to the applied force, can be calculated as:

$\sigma_{\mathrm{tbc}}=\frac{P}{2 h_{\mathrm{tbc}} w_{\mathrm{tbc}}}$

Substituting Eq. (2) into Eq. (1) yields

$G_{i} \approx \frac{P^{2}}{4}\left(\frac{1}{E_{\mathrm{sub}} h_{\mathrm{sub}} w_{\mathrm{sub}}^{2}}+\frac{1}{E_{\mathrm{tbc}} h_{\mathrm{tbc}} w_{\mathrm{tbc}}^{2}}\right)$

Because the maximum applied load obtained in the $P-u$ curve is believed to correspond to the onset of the TBC layer delamination from the substrate, the strain energy release rate for delamination cracking of the TBC layer from the substrate is determined using Eq. (3) with the maximum force, $P_{\max }$. Note that the instantaneous force drop behavior was neglected in determining the energy release rate using Eq. (3) although typical three or four load drops were observed after the maximum load (Fig. 4). Thus, the energy release rate determined with the peak load for initial delamination is the critical energy delamination criterion, i.e., toughness of interface. For the EB-PVD TBC system used, it is found that the measured critical strain energy release rates ranged from 60 to $90 \mathrm{~J} / \mathrm{m}^{2}$ using Eq. (3), with an average value of $\approx 70 \mathrm{~J} / \mathrm{m}^{2}$. With the present experimental method, these values are roughly the mode II strain energy release rates $\left(\psi=90^{\circ}\right)$ for either the TBC/bond coat interface or the TBC layer-dependent upon the observed fracture path selection. 
The mode II toughness of EB-PVD TBC has been measured at ambient temperature by both indentation testing [13] and wedge impression testing [14], and found to be of the order of $\Gamma_{\mathrm{II}}=60 \mathrm{~J} / \mathrm{m}^{2}$ when the mode II delaminations occurred predominantly at the $\mathrm{TGO} /$ bond coat interface $[13,14]$. The value obtained in the present measurements is higher than that mentioned above for the mode II toughness reported elsewhere [13,14] $\left(\Gamma_{\mathrm{II}}=60 \mathrm{~J} / \mathrm{m}^{2}\right)$. It is known that the critical strain energy release is linked to the properties of the bond coat, TGO, and TBC as well as their interface; properties such as the surface roughness and composition of the bond coat, thickness of TGO, residual stress at interface and physical properties of TBC. In particular, the residual stress at the interface and the Young's moduli used in determining the critical strain energy release value from Eq. (3) directly affect the measured value. However, these properties of TBC are not well characterized, and they could vary from specimen to specimen. In the present study, a possible reason for the higher toughness value that was obtained is correlated with observations of significant wear tracks on the post-test specimens (Fig. 5(a)). The influence of interfacial friction on the mode II toughness is documented in the literature. Mumm and Evans [14] showed that the mode II toughness value of $\mathrm{TGO} /$ bond coat interface $\left(\Gamma_{\mathrm{II}} 56 \mathrm{~J} / \mathrm{m}^{2}[14]\right)$ was lower than that of the oxide $\left(\Gamma_{\mathrm{II}} \geqslant 100 \mathrm{~J} / \mathrm{m}^{2}[15]\right)$. This low mode II toughness is attributable to the bending effect of the TGO/TBC bilayer as well as to shortening of the zone subject to friction over the full length of the delamination. The increasing effect of friction on the interface toughness was also reported by Reimanis et al. [25] after substantial crack extension for alumina scale bonded to Au substrate. They showed the value of toughness in the range of $10-55 \mathrm{~J} / \mathrm{m}^{2}$ for short cracks and $80-125 \mathrm{~J} / \mathrm{m}^{2}$ for cracks which have propagated just less than $1 \mathrm{~mm}$. In the present measurement, a larger frictional contribution relative to the indentation and wedge impression measurements was expected along the wake of the mode II delaminations because of significant frictional affects, as observed in SEM views of the post-test specimens (Fig. 5(a)). This large effect due to friction results from the longer contact zone subjected to friction as well as larger normal compressive stress acting on the TBC layer, as a result of fixing the TBC layer delamination edges during the barb testing and preventing bending of the TGO/TBC bilayer resulting from delamination.

\section{Concluding remarks}

A simple method of evaluating the shear mechanical properties of thermal barrier coating (TBC) systems has been developed. The technique is designed to evaluate crack growth resistance under essentially mode II loading conditions. The methodology has been applied to quantitatively measure the resistance to delamination of an EB-PVD TBC. The analysis has yielded estimates of the critical strain energy release rate for a TBC system. In the EB-PVD TBC system studied, the strain energy release rate was in a range of $60-90 \mathrm{~J} / \mathrm{m}^{2}$ with an average value of $70 \mathrm{~J} / \mathrm{m}^{2}$. The delamination crack propagated predominantly at the $\mathrm{TGO} / \mathrm{TBC}$, and $\mathrm{TGO} /$ bond coat interfaces. Although more detailed experimental studies and a refined analysis are needed to extract accurate values of coating and interfacial properties, the protocol described here is applicable to evaluate the interfacial mechanical response of various types of TBC systems.

\section{Acknowledgements}

The authors are grateful to Professor A.G. Evans, University of California, Santa Barbara, for valuable discussion. This work was partially supported by the New Energy and Industrial Technology Development Organization (NEDO) under the "Nano-Coating Project."

\section{References}

[1] Peters M, Leyens C, Schulz U, Kaysser WA. Adv Eng Mater 2001;3:193.

[2] Padture NP, Gell M, Jordan EH. Science 2002;296:280.

[3] Preauchat B, Drawin S. Surf Coat Technol 2001;146-147:94.

[4] Kim GM, Yanar NM, Hewitt EN, Pettit FS, Meier GH. Scripta Mater 2002;46:489.

[5] Schlichting KW, Padture NP, Jordan EH, Gell M. Mater Sci Eng A 2003;A342:120.

[6] Schulz U, Menzebach M, Leyens C, Yang YQ. Surf Coat Technol 2001;146-147:117.

[7] Mumm DR, Evans AG, Spitsberg IT. Acta Mater 2001;49:2329.

[8] Rabiei A, Evans AG. Acta Mater 2000;48:3963.

[9] Tolpygo VK, Clark DR, Murphy KS. Surf Coat Technol 2001;146-147:124.

[10] Sohn YH, Kim JH, Jordan EH, Gell M. Surf Coat Technol 2001;146-147:70.

[11] Tzimas E, Mullejans H, Peteves SD, Bressers J, Stamm W. Acta Mater 2000;48:4699.

[12] Wright PK. Mater Sci Eng A 1998;A245:191

[13] Vasinonta A, Beuth JL. Eng Fract Mech 2001;68:843.

[14] Mumm DR, Evans AG. Acta Mater 2000;48:1815.

[15] Clyne TW, Gill SC. J Therm Spray Technol 1996;5:401.

[16] Volinsky AA, Moody NR, Gerberich WW. Acta Mater 2002;50:441.

[17] Drory MD, Hutchinson JW. Proc Roy Soc Lond A 1996;452: 2319.

[18] Begley MR, Mumm DR, Evans AG, Hutchinson JW. Acta Mater 2000;48:3211.

[19] Yan J, Chen X, Karlsson AM. In preparation.

[20] Mumm DR, Faber KT. Acta Mater 1995;43:1259.

[21] Honda K, Kagawa Y. J Jpn Inst Metals 1994;58:91.

[22] Schulz U, Schmücker M. Mater Sci Eng A 2000;A276:1.

[23] Hutchinson JW, Suo Z. Adv Appl Mech 1992;29:63.

[24] Evans AG, He MY, Hutchinson JW. Prog Mater Sci 2001;46:249.

[25] Reimanis I, Dalgleish B, Evans A. Acta Metall Mater 1991; 39:3133. 